\section{Reducing Unplanned Extubations in the Pediatric ICU: Are We Seeing the Whole Picture?}

\section{To the Editor:}

We read with great interest the article by Tripathi et al $^{1}$ recently published in RESPIRATORY CARE assessing the impact of a quality-improvement project on the incidence of unplanned extubations. By carrying out this quality-improvement project, they were able to decrease the rate of unplanned extubations from 3.55 to 2.59 per 100 intubation days. The study opens debate regarding how to design a proper protocol to prevent unplanned extubation and for early prediction of high-risk patients; however, some aspects need to be discussed for adequate practical extrapolation.

Some of the definitions that the authors used in this study were different from those usually reported in the pediatric literature, making it difficult to interpret, validate, and generalize the results. The authors distinguish 2 forms of unplanned extubation: selfextubation, which is patient activity-related, and accidental, which involves no patient activity. Although the definition of self-extubation is widely used in adult studies, there is no previous study using such a definition for unplanned extubation in pediatric subjects. In fact, self-extubation is defined, in the available literature, as either deliberate or purposeful removal of the endotracheal tube by the patient her/himself. ${ }^{2}$ In contrast, accidental extubation is attributed to personnel's inappropriate manipulation of the tube during patient care or to a non-purposeful patient action. ${ }^{2}$ This distinction is of the utmost importance, since the types of unplanned extubations may differ significantly in terms of incidence, risk factors, and effects on patient outcomes. ${ }^{2}$ Given that neurocognitive development differs among children and is different throughout childhood, pediatric studies have not adopted the self-extubation definition when treating their data. Hence, for the sake of external validation, a more appropriate definition of pediatric unplanned extubation should have been used.

Furthermore, the authors found that 20 subjects $(55 \%)$ required re-intubation. Although pediatric studies define re-intubation as reinsertion of the endotracheal tube within a period of 24 or $48 \mathrm{~h},{ }^{3,4}$ the authors limited this period to $<1 \mathrm{~h}$. It would be interesting to know what the re-intu- bation rate would have been using the definition from the pediatric studies; however, making this happen has the potential to significantly change the analysis, given the small sample size of this study. Importantly, aligning the re-intubation definition would make it possible to better identify a high-risk population for worse outcomes, such as longer ICU stay, higher hospital costs, and increased rate of ventilator-associated pneumonia. ${ }^{5}$ Also, it is worth pointing out that the requirement for re-intubation is generally a marker of illness severity; however, there is no information about these data in the study.

Tripathi et $\mathrm{al}^{1}$ reported a total of 36 unplanned extubation events, 14 (38\%) of which were accidental. In addition, they found that of the total of 25 unplanned extubation subjects in the observation phase, $12(48 \%)$ were accidental extubation, whereas in the implementation phase, of the 11 unplanned extubation subjects, $2(11.8 \%)$ experienced accidental extubation. However, there is no reference to those subjects who had self-extubation. Considering that self-extubation subjects accounted for the majority of unplanned extubation events (22 of 36 subjects, $62 \%$ ), knowing their data as well as the circumstances involving the unplanned extubations is critical for the success of the quality-improvement program. The fact that self-extubation was more frequent than accidental in a sample of subjects that were mostly post-surgical $(71 \%)$ and ventilated short-term ( $42 \%$ with $<24 \mathrm{~h}$ ) makes us wonder if the timing for extubation was not adequate and if implementing interventions to approach missed opportunities for planned extubations is not worth it.

The authors used data from an interim analysis to establish the best practice policy for caring for intubated patients, focusing on high-risk environmental factors (weaning period, operating room-to-pediatric ICU transition) and patient-specific risk factors (children $<1$ y old, nursing procedures). After the implementation of their qualityimprovement program, the rate of unplanned extubations/100 intubation days dropped from 3.55 to 2.59 . Tripathi et $\mathrm{al}^{1}$ have not provided the statistical significance of this improvement. It is noteworthy to highlight that compared with contemporary qualityimprovement studies with 50-80\% improvement, $3,4,6$ a $27 \%$ improvement in the unplanned extubation rate is disappointing, since the final unplanned extubation rate re- mained higher than in other contemporary studies (range: 0.29-1.5/100 intubation days $)^{3,4,6-9}$ and also higher than the benchmark reported as acceptable in the literature $\left(<1 / 100\right.$ intubation days)..$^{10}$ In fact, the 2 largest studies in the pediatric literature have recently supported this recommendation achieving an incidence of $0.74^{11}$ and $0.77^{12}$ unplanned extubations per 100 intubation days.

Then again, and importantly, the authors only discussed the data for subjects with accidental extubation, which comprised only $38 \%$ of the total subjects who experienced unplanned extubations and, according to the authors, benefited the most from the quality-improvement program. Thus, since the impact of a quality-improvement program on the unplanned extubation rate was not remarkable, we can infer that those subjects who experienced self-extubation were the targets of most interest.

Because of the study design (before and after study), small sample size, and short period of study, it is not possible to rule out that the improvement in unplanned extubation rate was due to the Hawthorne effect, when the performance improvement is attributed to the simple fact that performance is being studied and not due to actual quality improvement. Ideally, a statistical process control method would have been used to investigate trends in the rate of unplanned extubation before the implementation of the program. Thus, stability during the post-intervention period is required to rule out extraneous trends and to convincingly document that the intervention is responsible for the performance. ${ }^{3}$

We have previously demonstrated significant improvement in unplanned extubation rates after a sustained collaborative effort from 2002 until 2007. ${ }^{3}$ During the last $8 \mathrm{y}$, we were able to maintain lower unplanned extubation rates, below the recommended benchmark, with ongoing reassessment, reinforcement, and reeducation applying interventions based on the PDSA (Plan, Do, Study, Act) cycle (Fig. 1). PDSA is a dynamic, continuous quality-improvement process. In this process, a quantifiable health-care problem (unplanned extubation), is approached with effective interventions targeting specific features of the problem/patients. Likewise, in a recent 9-y follow-up of unplanned extubation rate, Rachman et $\mathrm{al}^{8}$ showed that their quality-improvement program based on the PDSA model re- 


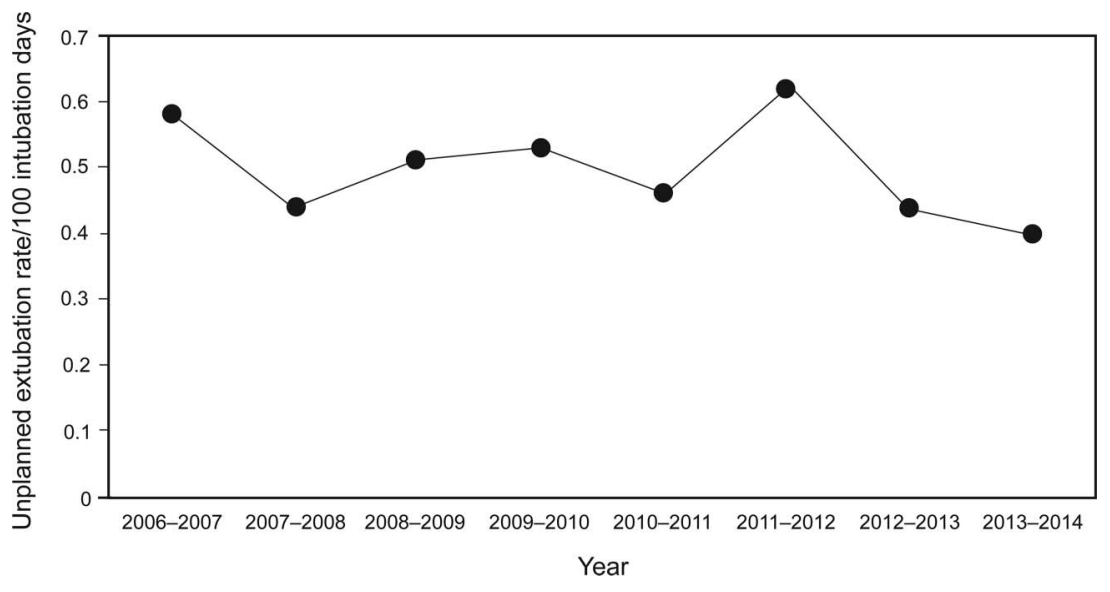

Fig. 1. Unplanned extubation rates in the pediatric ICU.

mained successful in decreasing unplanned extubations.

There was not, until recently, a multicenter study analyzing unplanned extubation risk factors. This year, Fitzgerald et $\mathrm{al}^{12}$ published a multi-center study involving 11 centers that found in children $<6$ y old $(0.83$ for $<6$ y vs $0.45 / 100$ intubation days for $\geq 6 \mathrm{y}, P=.001)$, inadequate sedation (odds ratio 9.1), loose or slimy endotracheal tube (odds ratio 10.4), a planned extubation in the next $12 \mathrm{~h}$ (odds ratio 2.3 ), and a floating nurse from another unit (odds ratio 3.8) were all risk factors for unplanned extubations. ${ }^{12}$

We have previously suggested a bundle approach to prevent unplanned extubation including both structure and process interventions. ${ }^{10}$ Nurse/patient ratios of $1: 1$ and the formation of a continuous quality-improvement team comprise the structural interventions. The process interventions include auditing practice, staff education and training, and standardization of routines as well as sedation protocols comprising routine sedation assessment and the use of targeted sedation. Although the efficacy and cost-effectiveness of such a bundle approach need to be determined, we believe that adopting one or more of these components may be helpful in decreasing the rate of unplanned extubations to a more acceptable level.

Paulo Sérgio Lucas da Silva MD MSc Pediatric Intensive Care Unit Department of Pediatrics Hospital do Servidor Píblico Municipal São Paulo, Brazil

Werther Brunow de Carvalho MD PhD
Pediatric Intensive Care Unit/Neonatal Intensive Care Unit Instituto da Criança Universidade de São Paulo São Paulo, Brazil

\section{Marcelo Cunio Machado Fonseca MD}

PhD

Department of Pediatrics Universidade Federal de São Paulo São Paulo, Brazil

The authors have disclosed no conflicts of interest.

DOI: $10.4187 /$ respcare. 04417

\section{REFERENCES}

1. Tripathi S, Nunez DJ, Katyal C, Ushay HM. Plan to have no unplanned: a collaborative, hospital-based quality-improvement project to reduce the rate of unplanned extubations in the pediatric ICU. Respir Care 2015; 60(8):1105-1112.

2. Kiekkas P, Aretha D, Panteli E, Baltopoulos GI, Filos KS. Unplanned extubation in critically ill adults: clinical review. Nurs Crit Care 2013;18(3):123-134.

3. da Silva PS, de Aguiar VE, Neto HM, de Carvalho WB. Unplanned extubation in a paediatric intensive care unit: impact of a quality improvement programme. Anaesthesia 2008;63(11):1209-1216.

4. Sadowski R, Dechert RE, Bandy KP, Juno J, Bhatt-Mehta V, Custer JR, et al. Continuous quality improvement: reducing unplanned extubations in a pediatric intensive care unit. Pediatrics 2004;114(3):628-632.

5. da Silva PS, Fonseca MC. Medical intelligence article: unplanned endotracheal extubations in the intensive care unit: systematic review, critical appraisal, and evi- dence-based recommendations. Anesth Analg 2012;114(5):1003-1014.

6. Kaufman J, Rannie M, Kahn MG, Vitaska M, Wathen B, Peyton C, et al. An interdisciplinary initiative to reduce unplanned extubations in pediatric critical care units. Pediatrics 2012;129(6):e1594-e1600.

7. Meregalli CN, Jorro Barón FA, D’Alessandro MA, Danzi EP, Debaisi GE. Impact of a quality improvement intervention on the incidence of unplanned extubations in a pediatric intensive care unit. Arch Argent Pediatr 2013;111(5):391-397.

8. Rachman BR, Mink RB. A prospective observational quality improvement study of the sustained effects of a program to reduce unplanned extubations in a pediatric intensive care unit. Paediatr Anaesth 2013; 23(7):614-620.

9. Rachman BR, Watson R, Woods N, Mink RB. Reducing unplanned extubations in a pediatric intensive care unit: a systematic approach. Int J Pediatr 2009;2009:820495. doi: 10.1155/2009/820495

10. Lucas da Silva PS, de Carvalho WB. Unplanned extubation in pediatric critically ill patients: a systematic review and best practice recommendations. Pediatr Crit Care Med 2010;11(2):287-294.

11. Kanthimathinathan HK, Durward A, Nyman A, Murdoch IA, Tibby SM. Unplanned extubation in a paediatric intensive care unit: prospective cohort study. Intensive Care Med 2015;41(7):1299-1306.

12. Fitzgerald RK, Davis AT, Hanson SJ. Multicenter analysis of the factors associated with unplanned extubation in the PICU. Pediatr Crit Care Med 2015;16(7):e217-e223.

\section{Reducing Unplanned Extubations in the Pediatric ICU: Are We Seeing the Whole Picture?-Reply}

\section{In Reply:}

We would like to thank Dr Da Silva and colleagues for their interest in our project report on interventions to reduce unplanned extubation in our pediatric ICU. ${ }^{1}$ We note with great interest their significant body of work in this important area of ICU safety.

The authors correctly note that the distinction between self-extubation and accidental extubation can be difficult in the pediatric population and, as a result, is not very widely reported in the literature. However, we believe that this distinction is important because different risk factors contribute to self-extubation and accidental extubation, and different interventions are needed to mitigate these risks. The authors are also correct in stating that the description of self-extubation ${ }^{2}$ comes from adult 